NBER WORKING PAPER SERIES

\title{
DISCOUNTING UNDER DISAGREEMENT
}

Geoffrey Heal

Antony Millner

Working Paper 18999

http://www.nber.org/papers/w18999

\author{
NATIONAL BUREAU OF ECONOMIC RESEARCH \\ 1050 Massachusetts Avenue \\ Cambridge, MA 02138 \\ April 2013
}

We are grateful for helpful comments from Martin Weitzman, Ken Arrow, Christian Gollier, Larry Karp, Geir Asheim, Reyer Gerlagh, Andreas Lange, and seminar participants at Paris Sorbonne, Hamburg, the TIGER forum '13, and the ASSA meetings '14. The views expressed herein are those of the authors and do not necessarily reflect the views of the National Bureau of Economic Research.

NBER working papers are circulated for discussion and comment purposes. They have not been peerreviewed or been subject to the review by the NBER Board of Directors that accompanies official NBER publications.

(C) 2013 by Geoffrey Heal and Antony Millner. All rights reserved. Short sections of text, not to exceed two paragraphs, may be quoted without explicit permission provided that full credit, including $\odot$ notice, is given to the source. 
Discounting under Disagreement

Geoffrey Heal and Antony Millner

NBER Working Paper No. 18999

April 2013, Revised February 2014

JEL No. D61,D99

\begin{abstract}
$\underline{\text { ABSTRACT }}$
A group of agents disagree about the appropriate inter temporal preferences to use when exploiting a common productive resource. They thus delegate decision making to a social planner who allocates consumption efficiently across heterogeneous individuals and over time. We define 'policy equivalent' representative agents as those agents whose optimal consumption plan reproduces the optimal aggregate consumption plan of the group, and find conditions that must be satisfied by all such representative preferences. We then show that any policy equivalent representative agent must have a rate of time preference that approaches the lowest rate in the population asymptotically. We characterize the term-structure of separable policy-equivalent preferences in the the case of common felicity functions, and show that if the felicity function is iso-elastic, and time preferences are gamma distributed, representative agents must have hyperbolic time preferences. This gives a normative significance to such preferences, which have hitherto been seen as an entirely behavioral phenomenon. Finally, we examine the temporal stability of representative preferences if the planner cannot commit to inter temporal allocations. We show that while consumption plans are not time consistent, they are renegotiation proof, and thus stable under proposals for change. We argue that this feature has normative appeal for public decision-making.
\end{abstract}

Geoffrey Heal

Graduate School of Business

616 Uris Hall

Columbia University

New York, NY 10027-6902

and NBER

gmh1@columbia.edu

Antony Millner

Grantham Research Institute

London School of Economics and Political Science

Houghton St

London, WC2A 2AE

United Kingdom

A.Millner@1se.ac.uk 


\section{Introduction}

Since the seminal contributions of Koopmans (1960), we have known that two plausible desiderata of dynamic choice - independence and stationarity (see e.g. Heal, 2005) - imply the standard exponential discounting model that forms the basis of dynamic welfare economics ${ }^{1}$. Decision makers with constant utility discount rates are time consistent, and this, in combination with the appeal of Koopmans' axioms, has provided the basis for the dominance of the exponential discounting model in normative applications.

In this paper we take the normative appeal of the exponential discounting model for granted, and assume that, at least for the purposes of evaluation of public policy, agents have time consistent preferences. However, like all preference representation results, Koopmans' provides us with a functional form for agents' preferences, but leaves the parameter values that enter their preferences - the actual value of the discount rate - unspecified. In order to operationalize the preference representation, we must look to the real world, and ask what agents' preferences actually are, or motivate a choice of discount rate by other normative considerations (Dasgupta and Heal, 1979; Arrow, 1999). Once we realize this, things become more murky. Whose discount rate should we choose? Are some discount rates more legitimate than others? What counts as a good argument for one value, rather than another?

We view an individual's choice of discount rate as an ethical primitive - it is a fundamental judgements about how she values realizations of welfare that are distant in time. Many things may inform this choice: ethical considerations of inter temporal equity, as well as judgements of whether the savings rate implied by her choice is desirable (Koopmans, 1969; Dasgupta, 2012). Although others may disagree with her choice, they cannot claim it is 'incorrect' - this is much the same as claiming that a preference for whisky over wine is incorrect. In this view, the problem of social discounting acquires a social choice dimension - there are many discount rates in the population, none is privileged over the others, and each should play a role in social decision making (Feldstein, 1964). There is abundant evidence for heterogeneous time preferences in the empirical literature (Frederick, Loewenstein and O'Donoghue, 2002). There is also substantial disagreement about the appropriate discount rate for normative applications, as demonstrated by the debate that followed the Stern Review of the Economics of Climate Change (Stern, 2007; Nordhaus, 2007; Weitzman, 2007), where the choice of discount rate was a crucial determinant of policy recommendations. Given this heterogeneity, and assuming the social choice approach has some democratic appeal, how should different discount rates be aggregated?

The standard dynamic welfare model deals with this question, if it deals with it at all, by fait accompli. We assume a representative agent (RA) whose preferences are themselves time consistent, and the problem boils down to motivating a choice for this agent's discount rate. This simplification, while undoubtedly attractive due to its tractability dividends, is often

\footnotetext{
${ }^{1} \mathrm{~A}$ few additional technical conditions are also needed, but these are less controversial.
} 
misleading. It has been recognized for some time that heterogeneous time preferences may lead the RA to have a non-constant discount rate. Gollier and Zeckhauser (2005) (henceforth GZ) consider a notion of representativeness based on distributive efficiency between heterogeneous agents who have access to a common non-storable income stream. They obtained an elegant result in this setup: The RA's discount rate is a weighted sum of the individuals' rates, where the weights depend on agents' time varying consumption allocations. Thus, the RA's discount rate is in general non-constant. GZ also show that when agents exhibit increasing tolerance for consumption fluctuations (or equivalently, decreasing absolute risk aversion), the RA's discount rate declines monotonically with time. Weitzman $(1998,2001)$ obtained qualitatively similar results in his analysis of the effect of uncertainty on the real (i.e. consumption) discount rate. The GZ result can thus be seen as a social choice theoretic analogue of Weitzman's analysis for the pure rate of time preference. Subsequently Jackson and Yariv (2012) have shown very generally that any social choice rule that aggregates individuals' preferences efficiently must be either dictatorial (i.e. coincide with exactly one person's preferences), or time inconsistent.

The GZ result is derived under the assumption that the group's aggregate income at each point in time is exogenously given and non-storable. In this paper we consider a more general setting in which the group manages a common productive resource endogenously over time. This context is, we feel, better suited to many of the interesting dynamic economic problems that require collective public choices to be made over policies. Examples include applications to Ramsey-style optimal growth models, and renewable and exhaustible resource management.

When aggregate consumption decisions are endogenous the notion of 'representative' preferences can take on new meanings. When the group's income is exogenous and non-storable, the only sense in which it is possible for an agent to represent the group is if its preferences are constructed so as to reproduce the welfare the group achieves for a given exogenous income stream. The RA's instantaneous welfare function is defined to coincide with the maximum of a weighted sum of individual utilities, subject to an income constraint. We shall refer to this notion of representativeness as welfare equivalence. When income is endogenous however, we may consider a different notion of representativeness, which we call policy equivalence. We say that an agent's preferences are policy equivalent if its optimal consumption plan coincides with the group's optimal aggregate consumption plan, so that the agent and the group make identical consumption-savings decisions. In the endogenous income case aggregate consumption decisions reflect the trade offs between different individuals in the social planner's allocation, and thus convey a great deal of relevant information. Policy equivalent preferences can be shown to give rise to the same real discount rate as the group would choose for itself. They are thus just as relevant as welfare equivalent preferences for the purposes of cost-benefit analysis. Policy equivalence is a weaker constraint on preferences than welfare equivalence - 
welfare equivalent preferences are policy equivalent, but the converse does not hold in general.

Our main result, derived in Section 2, provides a simple characterization of all policy equivalent preferences. It shows that policy equivalent time preferences can be expressed as a weighted sum of welfare equivalent time preferences and the productivity of the resource that provides the group's consumption. The weights on these two components vary with time, are endogenous, and correspond to a conversion factor between welfare equivalent and policy equivalent preferences for consumption smoothing. Using this characterization, we demonstrate that all policy equivalent preferences must converge to the lowest discount rate in the population asymptotically. This result makes a conceptual link between our approach, the analysis of long-run equilibria in economies with heterogeneous agents by Becker (1980), and a result of Weitzman (1998), which says that in the long run, certainty equivalent real discount rates tend to the 'lowest' rate.

The fact that policy equivalence is a weaker constraint on representative preferences than welfare equivalence affords us new freedom in our representation of the group's behavior. We demonstrate this in Section 3 by considering a case in which individuals share a common felicity function over consumption, but have heterogenous time preferences. Each individual thus has separable preferences, which obey Koopmans' stationarity axiom. In general however, the preferences of a welfare equivalent representative agent in the GZ framework will not be separable, and thus violate this axiom. By representing the group's behavior with policy equivalent preferences we can reinstate separability, thus maintaining consistency with the stationarity axiom, and capturing all the effects of discount rate heterogeneity in a separable discount factor. This has intuitive appeal - since agents disagree only about the discount rate in this scenario it is attractive to capture the effects of disagreement in a modified collective discount rate, while leaving the mutually agreed felicity function unchanged. We examine this case in detail, demonstrating the relationship between welfare equivalent and separable policy equivalent preferences, and characterizing their term structures.

A very special case arises when agents' common felicity function is iso-elastic. In this case welfare equivalent and separable policy equivalent preferences are identical, and give rise to representative agents that have separable preferences. Since this choice of felicity function is common in applications, we study its properties in more detail, and demonstrate that if we assume, following Weitzman (2001), that agents' rates of time preferences are gamma distributed, the group's discount rate will be a hyperbolic function of time. This allows us to reinterpret the classic behavioral models of hyperbolic discounting (e.g. Loewenstein and Prelec, 1992; Laibson, 1997; Dasgupta and Maskin, 2005) in a normative light.

Finally, we examine the temporal stability of representative preferences if the social planner cannot commit to her initial allocations in Section 4. In this case the fact that agents' time preferences are heterogeneous gives rise to a time consistency problem, which is re- 
flected in the time varying discount rates of representative agents ${ }^{2}$. The presence of multiple heterogeneous individuals may nevertheless act as a stabilizing force on the group's initial consumption plans. We observe that if the group can agree to implement an efficient plan in the first period, that plan will be renegotiation-proof, and thus stable under a rule that requires changes in allocations to be unanimously agreed upon. Thus, although initial allocations are not time consistent if the social planner cannot commit to her plans, they may nevertheless be implemented if we allow for this weaker concept of temporal stability. We argue that this formulation has attractive normative features. Section 5 concludes.

\section{Related literature}

A model of heterogeneous preferences and endogenous resource management has been studied by Li and Löfgren (2000). Our work is differentiated from their approach in that we consider consumption derived from a common resource which may be differentially allocated, whereas consumption in their model is a public good. The social planner in our model thus decides on both aggregate consumption and the consumption share of each individual endogenously. Models of heterogeneous preferences have also been investigated in financial economics, where the focus is on private investment decisions and the effects of heterogeneity on market equilibria, interest rates, and risk premia (e.g. Dumas, 1989; Lengwiler, 2005; Hara, 2009; Cvitanić et al., 2012). This work focuses on positive questions and private choices, whereas our work deals with normative questions and public choices. In addition, all of this work assumes, as is common in financial economics, that consumption paths are exogenous. Finally, the properties of the welfare equivalent representation of a heterogeneous group's preferences with exogenous consumption have been examined by Wilson (1968); Gollier and Zeckhauser (2005); Nocetti, Jouini and Napp (2008); Jouini, Marin and Napp (2010). Our work uses the endogeneity of aggregate consumption decisions to investigate policy equivalent preferences. This gives us much greater freedom in representing the group's behavior.

\section{Representative time preferences}

\subsection{The social planner's problem}

Consider a set of agents, indexed by $i$. Each of the agents is assumed to have additive, time consistent preferences. Thus, the utility of agent $i$ from consumption $c_{t i}$ realized at time $t$ in the future is $U_{i}\left(c_{t i}\right) e^{-\rho_{i} t}$, where $U_{i}(\cdot)$ is the agent's felicity function, and $\rho_{i}$ is its pure rate of time preference. The group of agents has access to a common managed 'resource' $S$, which is the source of their income. We can think of $S$ as physical or natural capital. The resource has

\footnotetext{
${ }^{2}$ This issue is present in all models of efficient preference aggregation by a social planner, including e.g. Gollier and Zeckhauser (2005).
} 
a natural growth function $F(S)$, which we assume to be a concave function with $F^{\prime}(0) \geq 0$. Otherwise, we keep the form of $F(S)$ unspecified at this stage. This model accommodates a wide class of stock management problems, including neoclassical growth models, and models of renewable and exhaustible resource management.

We assume that a social planner commits to exploiting the resource $S$ so that allocations of consumption across individuals maximize social welfare, which is taken to be a Paretoweighted sum of individuals' welfare. Consumption allocations are thus efficient. The social planner's allocation problem is:

$$
\max _{c_{t i}} \sum_{i} w_{i}\left(\int_{0}^{\infty} U_{i}\left(c_{t i}\right) e^{-\rho_{i} t} d t\right) \quad \text { s.t. } \quad \dot{S}=F(S)-\sum_{i} c_{t i}
$$

where the welfare weights $w_{i} \in(0,1)$ satisfy $\sum_{i} w_{i}=1$, and the initial value of the stock is $S(0)=S_{0}$.

In addition to the standard assumptions $U_{i}^{\prime}>0, U_{i}^{\prime \prime}<0$ on the felicity functions, we also assume that $\lim _{c \rightarrow 0} U_{i}^{\prime}(c)=\infty$. This ensures that the solution to the planner's control problem is interior. The curvature of $U_{i}(c)$ measures agents' tolerance for consumption fluctuations. It will prove useful in what follows to have a way of measuring this important aspect of agents' intertemporal preferences. To this end we define the Absolute Tolerance for Consumption Fluctuations associated with a felicity function $U_{i}(c)$ as:

$$
T^{i}(c):=-\frac{U_{i}^{\prime}(c)}{U_{i}^{\prime \prime}(c)}
$$

This quantity tells us about agents' consumption smoothing preferences. The smaller is $T^{i}$, the more the agent cares about smoothing its consumption over time. $T^{i}(c)$ is the inverse of the coefficient of absolute risk aversion, though uncertainty will play no role in our analysis. The familiar assumption of decreasing absolute risk aversion corresponds to the assumption that $T^{i}(c)$ is an increasing function. This has an equally direct interpretation in our context: the higher is $c$, the more tolerant agents are towards small additive consumption fluctuations. We will assume this condition in what follows. The requirement that marginal felicity be unbounded at $c=0$ places restrictions on the behavior of $T^{i}(c)$ near zero. We show in Appendix A that it implies

$$
\lim _{c \rightarrow 0} T^{i}(c)=0
$$

This condition will be useful later on.

The Hamiltonian for the social planner's problem is:

$$
H=\sum_{i} w_{i} U_{i}\left(c_{t i}\right) e^{-\rho_{i} t}+\lambda\left(F(S)-\sum_{i} c_{t i}\right),
$$


where $\lambda$ is the shadow price of the resource. Since the Hamiltonian is concave, the necessary conditions of the Pontryagin Maximum Principle are sufficient for an optimum, and the optimum is unique. The necessary conditions are:

$$
\begin{aligned}
\frac{\partial H}{\partial c_{t i}} & =0 \Rightarrow \lambda=e^{-\rho_{i} t} U_{i}^{\prime}\left(c_{t i}\right) w_{i} \\
\frac{\partial H}{\partial S} & =-\dot{\lambda} \Rightarrow-\dot{\lambda}=F^{\prime}(S) \lambda \\
\lim _{t \rightarrow \infty} \lambda S & =0
\end{aligned}
$$

Hence along an optimal trajectory, the stock evolves according to the dynamical system

$$
\begin{aligned}
& \dot{\lambda}=-\lambda F^{\prime}(S) \\
& \dot{S}=F(S)-\sum_{i}\left(U_{i}^{\prime}\right)^{-1}\left(e^{\rho_{i} t} \lambda / w_{i}\right)
\end{aligned}
$$

and at each point in time consumption is allocated so that

$$
c_{t i}=\left(U_{i}^{\prime}\right)^{-1}\left(e^{\rho_{i} t} \lambda / w_{i}\right)
$$

The initial condition on the shadow price will be part of the solution. Denote the optimal aggregate consumption path associated with the group's problem by

$$
C_{t}^{*}=\sum_{t} c_{t i}
$$

and the associated optimal path of the resource stock by $S_{t}^{*}$.

\subsection{Representative agents and the group's discount rate}

We are interested in defining a representative agent whose preferences capture the heterogeneity in the population, and its effects on the group's behavior. There are two natural definitions of representativeness in our setup: welfare equivalence and policy equivalence. Both notions require us to specify an instantaneous utility function for the representative agent which depends only on the group's aggregate consumption, and time.

Welfare equivalent preferences are constructed so that the welfare of the representative agent coincides with that of the group at each point in time. Suppose that the group's aggregate consumption at time $t$ is $C_{t}$. We know that aggregate consumption $C_{t}$ must be efficiently distributed. Thus the instantaneous welfare the group will realize at time $t$ is captured by the following function:

$$
V\left(C_{t}, t\right):=\max _{\tilde{c}_{t i}} \sum_{i} w_{i} U_{i}\left(\tilde{c}_{t i}\right) e^{-\rho_{i} t} \text { s.t. } \sum_{i} \tilde{c}_{t i}=C_{t}
$$


The elasticities of $\partial V\left(C_{t}, t\right) / \partial C_{t}$ with respect to time and aggregate consumption yield the group's welfare equivalent time preferences, and the inverse of its absolute tolerance for consumption fluctuations, respectively. Gollier and Zeckhauser (2005) derive expressions for these welfare equivalent quantities. They show that at the optimal allocation of the $\tilde{c}_{t i}$ we have

$$
\begin{aligned}
\rho^{V}\left(C_{t}, t\right) & :=-\frac{V_{t C}}{V_{C}}=\frac{\sum_{i} \rho_{i} T^{i}\left(\tilde{c}_{t i}\right)}{\sum_{i} T^{i}\left(\tilde{c}_{t i}\right)} \\
T^{V}\left(C_{t}, t\right) & :=-\frac{V_{C}}{V_{C C}}=\sum_{i} T^{i}\left(\tilde{c}_{i}\right) .
\end{aligned}
$$

Notice that welfare equivalent preferences are defined without knowledge of the process that generates the group's consumption - we simply treat $C_{t}$ as an exogenously given income stream. Thus, while welfare equivalent preferences capture the realized value of consumption, they do not capture the effects of preference heterogeneity on the endogenous aggregate consumption decisions of the group.

Policy equivalent preferences are constructed so that the representative agent's optimal consumption plan coincides with the social planner's equilibrium. We now aim to characterize such preferences. Consider a representative agent (RA) with instantaneous utility function $R(C, t)$. We impose no restrictions on $R(C, t)$ except that it be increasing and concave in $C$, and satisfy $\lim _{C \rightarrow 0} \frac{\partial R(C, t)}{\partial C}=\infty$ for all $t$. This RA will solve:

$$
\max _{C_{t}} \int_{0}^{\infty} R\left(C_{t}, t\right) d t \text { s.t. } \dot{S}_{t}=F\left(S_{t}\right)-C_{t}
$$

We want to characterize all preferences $R(C, t)$ that are consistent with the group's aggregate consumption path, as defined by the solution to the dynamical system (8-9). Define

$$
\begin{gathered}
\rho^{R}(C, t)=-\frac{R_{C t}}{R_{C}} \\
T^{R}(C, t)=-\frac{R_{C}}{R_{C C}},
\end{gathered}
$$

where subscripts denote partial derivatives. Denote the optimal paths of consumption and the resource stock associated with preferences $R(C, t)$ by $C_{t}^{R}$ and $S_{t}^{R}$ respectively. An application of the Maximum Principle shows that along the equilibrium path associated with the RA's problem (15), we must have:

$$
\rho^{R}\left(C_{t}^{R}, t\right)+\frac{\dot{C}_{t}^{R}}{T^{R}\left(C_{t}^{R}, t\right)}=F^{\prime}\left(S_{t}^{R}\right)
$$

Our task is to find conditions on the preference functions $\rho^{R}$ and $T^{R}$ that must be satisfied if the representative agent's consumption path is to emulate that of the social planner. In order to do this we return to considering the group's optimum. Differentiate (5) with respect 
to $t$, and substitute into (6), to find

$$
\dot{c}_{t i}=\left(F^{\prime}\left(S^{*}\right)-\rho_{i}\right) T^{i}\left(c_{t i}\right)
$$

Use (13-14), and sum (19) over $i$ to find

$$
\begin{aligned}
\dot{C}_{t}^{*}=\sum_{i} \dot{c}_{t i} & =\left(F^{\prime}\left(S^{*}\right)-\frac{\sum_{i} \rho_{i} T^{i}\left(c_{t i}\right)}{\sum_{i} T^{i}\left(c_{t i}\right)}\right) \sum_{i} T^{i}\left(c_{t i}\right) \\
& =\left(F^{\prime}\left(S^{*}\right)-\rho^{V}\left(C_{t}^{*}, t\right)\right) T_{V}\left(C_{t}^{*}\right)
\end{aligned}
$$

Note that we've used the properties of the function $V\left(C_{t}, t\right)$, defined in (12), to re-express some of the terms in the evolution equation for the group's aggregate consumption.

Our identifying assumption is that the RA's preferences give rise to the same optimal consumption plan as the group:

$$
\forall t, \quad C_{t}^{R}=C_{t}^{*}
$$

We need to impose this condition on the equation that determines the RA's equilibrium path, i.e. (18). Differentiating (21) with respect to time, and making use of (20), we see that imposing (21) on (18) leads to the following necessary condition on the preference functions $\rho^{R}$ and $T^{R}$ :

$$
\rho^{R}\left(C_{t}^{*}, t\right)=\left[1-\frac{T^{V}\left(C_{t}^{*}, t\right)}{T^{R}\left(C_{t}^{*}, t\right)}\right] F^{\prime}\left(S_{t}^{*}\right)+\left[\frac{T^{V}\left(C_{t}^{*}, t\right)}{T^{R}\left(C_{t}^{*}, t\right)}\right] \rho^{V}\left(C_{t}^{*}, t\right) .
$$

We can write this slightly more succinctly by defining

$$
X^{R}(C, t):=\frac{T^{V}(C, t)}{T^{R}(C, t)} .
$$

Hence our necessary conditions are

$$
\rho^{R}\left(C_{t}^{*}, t\right)=\left(1-X^{R}\left(C_{t}^{*}, t\right)\right) F^{\prime}\left(S_{t}^{*}\right)+X^{R}\left(C_{t}^{*}, t\right) \rho^{V}\left(C_{t}^{*}, t\right)
$$

The functions $F^{\prime}\left(S_{t}^{*}\right), \rho^{V}\left(C_{t}^{*}, t\right)$, and $T^{V}\left(C_{t}^{*}, t\right)$ are all exogenously given. Note that we need this relationship between the preference functions $\rho^{R}$ and $T^{R}$ (or $X^{R}$ ) to hold only on the group's equilibrium path $C_{t}^{*}$, and for no other paths. This ensures that only the group's equilibrium path satisfies the RA's equilibrium conditions.

The fact that (24) is required to hold only on the group's equilibrium path illustrates the degeneracy in the set of preferences that are able to replicate the group's consumption behavior. We are free to specify the values of $R\left(C_{t}, t\right)$ on off-equilibrium paths as we choose, provided we respect (24), and the relevant smoothness and concavity properties necessary for making the solution to the RA's optimization problem unique. The off-equilibrium values of 
$R\left(C_{t}, t\right)$ are not however relevant for understanding the group's discounting behavior - we care only about preferences on the equilibrium path, as this is the path that is in fact realized.

We will say that preferences $R(C, t)$ that satisfy (24) are policy equivalent to the group's equilibrium. It is trivially true that any policy equivalent preference must yield the correct schedule for the group's real (i.e. consumption) discount rate. The value of a marginal change in consumption at time $t$ to a policy equivalent representative agent in today's consumption units is just $\lambda^{R}(t) / \lambda^{R}(0)$, where $\lambda^{R}$ is the shadow price corresponding to the representative preferences $R$. The real discount rate is the rate of change of the value of a marginal change in consumption: $-\frac{d}{d t}\left(\frac{\lambda^{R}(t)}{\lambda^{R}(0)}\right)=F^{\prime}\left(S_{t}^{R}\right)=F^{\prime}\left(S_{t}^{*}\right)$. The first equality follows from the representative agent's first order conditions, and the second from the fact that, by construction, the evolution of the resource stock is the same under the representative agent and the group itself. Any policy equivalent agent will thus discount marginal changes in consumption in the same manner as the group. Thus, although there are many possible policy equivalent preferences, they all give rise to the same real discount rate, and hence the same cost-benefit rules for the evaluation of marginal projects.

Policy equivalence is a weaker constraint on preferences than welfare equivalence. In fact, welfare equivalent preferences are policy equivalent, but the converse does not hold in general. To see this note that the planner's optimization problem can be written as:

$$
\begin{aligned}
& \max _{c_{t i}} \sum_{i} w_{i}\left(\int_{0}^{\infty} U_{i}\left(c_{t i}\right) e^{-\rho_{i} t} d t\right) \text { s.t. } \dot{S}=F(S)-\sum_{i} c_{t i} \\
\equiv & \max _{C_{t}} \int_{0}^{\infty} \max _{c_{t i}}\left(\sum_{i} w_{i} U_{i}\left(c_{t i}\right) e^{-\rho_{i} t} \text { s.t. } \sum_{i} c_{t i}=C_{t}\right) d t \text { s.t. } \dot{S}=F(S)-C_{t} \\
\equiv & \max _{C_{t}} \int_{0}^{\infty} V\left(C_{t}, t\right) d t \text { s.t. } \dot{S}=F(S)-C_{t}
\end{aligned}
$$

The last of these formulations is of the same form as (15), and clearly the equilibria of the first and last formulations must coincide. Thus welfare equivalent preferences are policy equivalent. It is evident from (24) that for any $X^{R}\left(C_{t}, t\right) \neq 1, \rho^{R}\left(C_{t}, t\right) \neq \rho^{V}\left(C_{t}, t\right)$, and so policy equivalent preferences need not be welfare equivalent.

The choice between the two notions of representativeness is in part a matter of convenience for the problem at hand, and in part a question of which properties one wishes to ensure representative preferences possess. Welfare equivalent preferences are easier to transfer between different problems as they do not require knowledge of the production function $F(S)$, but in general they give rise to non-separable utility functions that may be difficult to interpret. Policy equivalent preferences are in general context dependent but, owing to the additional freedom in their specification, can be chosen to be separable, as we demonstrate in Section 3 below. Next however, we show that although the set of policy equivalent preferences may be large, they are all tightly constrained in the long run. 


\subsection{The steady state and the long-run discount rate for policy equivalent preferences}

We now show that at a steady state $S_{\infty}$ of the dynamical system (8-9), all policy equivalent preferences $R(C, t)$ give the same answer for the RA's discount rate: the long run discount rate coincides with the lowest rate in the population, regardless of the exact form of the representative agent's preferences.

To see this notice that when the resource stock is stationary, i.e. $\dot{S}=0$, we must have

$$
F(S)-\sum_{i} c_{t i}=0
$$

Since this equation must hold for all times at the steady state, we can differentiate it with respect to time, and set the result to zero:

$$
F^{\prime}(S) \dot{S}-\sum_{i} \dot{c}_{t i}=-\left(F^{\prime}(S)-\rho^{V}\right) \sum_{i} T^{i}\left(c_{t i}\right)=0
$$

where we've used (20). There are two possibilities, either $F^{\prime}(S)=\rho^{V}$, or $\sum_{i} T^{i}\left(c_{t i}\right)=0$. Assuming a non-trivial steady state (i.e. $S \neq 0$ ) at least one agent's consumption will be positive, thus we must have $F^{\prime}(S)=\rho^{V}$, and hence from (24), we conclude that

$$
\left.\rho^{R}\right|_{S=S_{\infty}}=\rho^{V}
$$

The following proposition determines how consumption is allocated at the steady state, and hence allows us to infer the long run discount rate for all policy equivalent preferences in general via (27).

Proposition 1. Let $L$ be the index of the agent with the lowest discount rate, $\rho_{L}$. Assume that the equation $F^{\prime}(S)=\rho_{L}$ has a solution ${ }^{3}$ in $S$. Then the only nontrivial steady state $S_{\infty}$ of our model is given by

$$
\begin{aligned}
& S_{\infty}=\left(F^{\prime}\right)^{-1}\left(\rho_{L}\right) \\
& c_{\infty i}=\left\{\begin{array}{cc}
0 & i \neq L \\
F\left(S_{\infty}\right) & i=L
\end{array}\right.
\end{aligned}
$$

and the steady state is a saddle point of the dynamical system (8-9). Moreover, for all preferences $R(C, t)$ that replicate the group's behavior,

$$
\lim _{t \rightarrow \infty} \rho^{R}\left(C_{t}^{*}, t\right)=\rho_{L}
$$

\footnotetext{
${ }^{3}$ This is guaranteed by continuity of $F^{\prime}(S)$ if $\lim _{S \rightarrow 0} F^{\prime}(S)>\rho_{L}, \lim _{S \rightarrow \infty} F^{\prime}(S)<\rho_{L}$. The solution must be unique by our assumption that $F$ is concave.
} 
Proof. See Appendix B.

The fact that the steady state is a saddle point, and the only non-trivial steady state of the system (8-9), means that we know that the optimal trajectory of the system must be a saddle path. In general, the saddle path will be monotonic, i.e. the state variable $S$ will

approach its steady state value $S_{\infty}$ monotonically from its initial value $S_{0}$ (see e.g. Kamien and Schwartz, 1991).

Proposition 1 parallels a result obtained by Becker (1980), who showed that in the longrun steady state of a competitive economy where agents have heterogeneous time preferences, all consumption accrues to the most patient individual. The endogeneity of the group's consumption path in our model has thus allowed us to obtain an analogue of the results of Weitzman (1998), who shows that uncertainty about the real discount rate implies that in the long run the certainty equivalent discount rate approaches the lowest rate in the long run. In our case, disagreement over the pure rate of time preference implies that the group's rate of time preference approaches the lowest rate in the population asymptotically, regardless of how we choose to represent its behavior.

\section{Common felicity functions}

In order to demonstrate the flexibility that the policy equivalent approach to representative preferences affords us, and investigate the properties of the schedule of policy equivalent time preferences, we now consider a case in which all agents agree on the felicity function, $U_{i}(c)=U(c)$ (with $U^{\prime}>0, U^{\prime \prime}<0, \lim _{c \rightarrow 0} U^{\prime}(c)=\infty$ ), but have different rates of time preference $\rho_{i}$. In this case it is natural to seek a representative agent whose preferences reflect the source of disagreement between the agents. In particular, since the agents agree on the felicity function but disagree on time preferences, we might expect the representative agent to share their assessment of the value of consumption, but to have a modified discount rate that accounts for the heterogeneity in their time preferences. Unfortunately this option is not open to us if we restrict ourselves to welfare equivalent representative preferences. Welfare equivalent preferences are in general non-separable, and thus we cannot use the common felicity function $U(c)$ when representing the group's behavior.

If, however, we consider policy equivalent preferences, we have the freedom to preserve the separability of representative preferences, and hence to utilize $U(c)$. Begin by defining separable policy equivalent representative preferences as follows:

$$
R\left(C_{t}, t\right)=U\left(C_{t}\right) e^{-\rho^{*}(t) t}
$$

We can use the condition (24), and the definitions (13-14) to immediately write down the 
conditions that $\rho^{*}(t)$ must satisfy in order to ensure policy equivalence:

$$
\begin{aligned}
\rho^{*}(t) & =\left(1-X^{U}\left(C_{t}^{*}, t\right)\right) F^{\prime}\left(S_{t}^{*}\right)+X^{U}\left(C_{t}^{*}, t\right) \rho^{U}\left(C_{t}^{*}, t\right), \\
X^{U}\left(C_{t}, t\right) & :=\frac{\sum_{i} T\left(c_{t i}\right)}{T\left(\sum_{i} c_{t i}\right)}, \\
\rho^{U}\left(C_{t}, t\right) & :=\frac{\sum_{i} \rho_{i} T\left(c_{t i}\right)}{\sum_{i} T\left(c_{t i}\right)}
\end{aligned}
$$

where $T(c)=-U^{\prime} / U^{\prime \prime}$ is the absolute tolerance of consumption fluctuations for the common felicity function $U(c)$.

A natural question to ask is whether we can find conditions that determine the qualitative properties of the term structure of the representative discount rate $\rho^{*}(t)$, and its relationship to the welfare equivalent discount rate $\rho^{U}(t)$. In general, the derivative of $\rho^{*}(t)$ with respect to time is a complex function. We have however obtained the following proposition:

\section{Proposition 2. Suppose that}

1. $T$ is increasing and convex.

2. $T / T^{\prime}$ is convex.

3. If there is a non-trivial steady state: $S_{0}<S_{\infty}$.

Then $\rho^{*}(t)$ is monotonically declining in time. $\rho^{*}(t) \geq \rho^{U}(t)$ if there is a non-trivial steady state, and $\rho^{*}(t) \leq \rho^{U}(t)$ if $S$ is an exhaustible resource (i.e. $F(S)=0$ ).

Proof. See Appendix C.

As we show in Appendix $\mathrm{C}$, the conditions on $T$ in this proposition must hold locally at $c=0$, we have simply converted them to global conditions. An example of a class of functions that satisfies these conditions is $T(c)=K c^{\alpha}$, where $K>0, \alpha \geq 1$. The familiar case of isoelastic felicity functions corresponds to $\alpha=1$, and will be examined in detail in the following section. The condition on $S_{0}$, i.e. that the initial value of $S$ be below its steady state value, is natural in most applications where the resource $S$ is 'renewable', including neoclassical growth models and renewable resource management problems. When the resource is exhaustible, i.e. $F(S)=0$, the only steady state is $S=0$. We show in Appendix $\mathrm{C}$ that the conditions on $T(c)$ in the proposition still guarantee a monotonically declining discount rate in this case.

\subsection{Parametric examples}

In this section we specialize to specific parametric cases for the common felicity function $U(c)$ and the resource's growth function $F(S)$. This will allow us to investigate quantitative properties of policy equivalent time preferences, thus moving beyond the qualitative results of Proposition 2. 


\subsubsection{Iso-elastic felicity and Gamma discounting}

The evolution of the policy-equivalent discount rate (32) is in general complex, as it depends on the evolution of the dynamical system (8-9). Here we focus on the special case of iso-elastic felicity functions. This serves two purposes: iso-elastic felicity is a widely used functional form in applications, and analytic solutions for $\rho^{*}(t)$ are possible in this case.

It's clear from the definition of $X$ in (33), that $X=1$ if $T(c)$ is a linear function. This case corresponds to the work-horse case of an iso-elastic felicity function. Specifically,

$$
U(c)=\frac{c^{1-\eta}}{1-\eta} \Rightarrow T(c)=\frac{c}{\eta}
$$

We can use this explicit functional form to calculate the inverse of marginal felicity, and hence (10) determines the values of the agents' consumption:

$$
c_{t i}=\left(\frac{w_{i} e^{-\rho_{i} t}}{\lambda(t)}\right)^{\frac{1}{\eta}}
$$

Substituting (35-36) and $X=1$ into (24) shows that in this special case:

$$
\rho_{\eta}^{*}(t)=\frac{\sum_{i} \rho_{i}\left(w_{i} e^{-\rho_{i} t}\right)^{\frac{1}{\eta}}}{\sum_{i}\left(w_{i} e^{-\rho_{i} t}\right)^{\frac{1}{\eta}}} .
$$

This result holds for any stock dynamics $F(S)$ - the shadow price has fallen out of the expression for $\rho^{*}(t)$. Thus, in this case the policy-equivalent separable discount rate (32) reduces to welfare-equivalent formula (13). This is the only felicity function for which welfareequivalent preferences are separable, and thus the two notions of representativeness coincide in this case. It is straightforward to verify that $\rho_{\eta}^{*}(t)$ declines monotonically to the lowest rate $\rho_{L}$, in agreement with Propositions 1 and 2 .

The group's discount rate $\rho_{\eta}^{*}(t)$ is a weighted sum of the individuals' discount rates,

with time dependent weights $y_{i t}:=\left(w_{i} e^{-\rho_{i} t}\right)^{\frac{1}{\eta}}$. We can find an analytic expression for the dependence of $\rho^{*}(t)$ on the distribution of individual preferences in a special case for the weights $w_{i}$. Take the continuum limit of $(37)$, and let $w_{i} \rightarrow w(\rho)$ be the probability density of discount rate $\rho$. Assume, following Weitzman (2001), that $w(\rho)$ is Gamma distributed with shape and scale parameters $k, \theta>0$. This implies:

$$
w(\rho) \propto \rho^{k-1} e^{-\frac{\rho}{\theta}} .
$$

Thus the time dependent weights $y_{i t}$ become

$$
y_{i t} \rightarrow y_{t}(\rho) \propto \rho^{\left(\frac{k-1}{\eta}+1\right)-1} e^{-\frac{1}{\eta}\left(\frac{1}{\theta}+t\right) \rho} .
$$


These are also Gamma distributed, with modified parameters $\left(k^{\prime}, \theta^{\prime}\right)=\left(\frac{k-1}{\eta}+1, \eta /\left(\theta^{-1}+t\right)\right)$. The Gamma distribution is not strictly defined for $k^{\prime}<0$, but one can show from (37) that $\rho_{\eta}^{*}(t)=0$ in this case. When $k^{\prime} \geq 0$, using the fact that the mean of the Gamma distribution with parameters $\left(k^{\prime}, \theta^{\prime}\right)$ is just $k^{\prime} \theta^{\prime}$, we have

$$
\rho_{\eta}^{*}(t)=\frac{k-1+\eta}{t+\frac{1}{\theta}}
$$

Now noting that the mean $\mu$ and variance $\sigma^{2}$ of the Gamma distribution are related to $k$ and $\theta$ through $k=\frac{\mu^{2}}{\sigma^{2}}$, and $\theta=\frac{\sigma^{2}}{\mu}$, we find

$$
\rho_{\eta}^{*}(t)=\left\{\begin{array}{cl}
\frac{\mu+\left(\sigma^{2} / \mu\right)(\eta-1)}{1+\sigma^{2} t / \mu} & \eta \geq 1-\frac{\mu^{2}}{\sigma^{2}} \\
0 & \eta<1-\frac{\mu^{2}}{\sigma^{2}}
\end{array}\right.
$$

Note that $\rho_{\eta}^{*}$ is non-decreasing in $\eta$. We expect this since the larger is $\eta$, the less tolerant agents are of consumption fluctuations - this makes the group's consumption decisions more myopic. $\rho_{\eta}^{*}$ is also non-decreasing in $\mu$ - the larger is the average pure rate of time preference, the larger is the group's rate of time preference. Interestingly,

$$
\operatorname{sgn}\left(\frac{\partial \rho_{\eta}^{*}}{\partial \sigma^{2}}\right)=\operatorname{sgn}\left(\frac{\eta-1}{\mu}-t\right)
$$

Thus the direction of the effect of an increase in the variance of time preferences varies with time when $\eta>1$. For $t<(\eta-1) / \mu$, the group's discount rate increases, and for $t>(\eta-1) / \mu$ it decreases if we increase $\sigma^{2}$. Also, $\lim _{\sigma^{2} \rightarrow 0} \rho_{\eta}^{*}(t)=\mu$ and $\lim _{\sigma^{2} \rightarrow \infty} \rho_{\eta}^{*}(t)=(\eta-1) / t$ (for $\eta>1$ ). The time preferences of high variance groups look more 'hyperbolic', while those of low variance groups look more like the constant discount rate model. Figure 1 illustrates this dependence.

The functional form for $\rho_{\eta}^{*}(t)$ in (41) corresponds to the hyperbolic discounting model, familiar from applications in behavioral economics (e.g. Loewenstein and Prelec, 1992; Laibson, 1997). Our analysis suggests an alternative interpretation for this model of a representative agent's time preferences. If social planners can commit to efficient dynamic distributions of consumption, agents have common iso-elastic felicity functions, and time preferences are gamma distributed, then the planner's consumption plan is equivalent to that of a representative agent with hyperbolic time preferences. This holds for any stock dynamics, and thus allows us to interpret the hyperbolic discounting model normatively, rather than behaviorally. A crucial assumption of this result is that the social planner commits to her plans for all time. While this may be an appropriate assumption in normative work, it is not in behavioral applications. Laibson (1997) and Barro (1999) explore the consequences of lack of commitment in behavioral models. We return to this issue below in Section 4. 


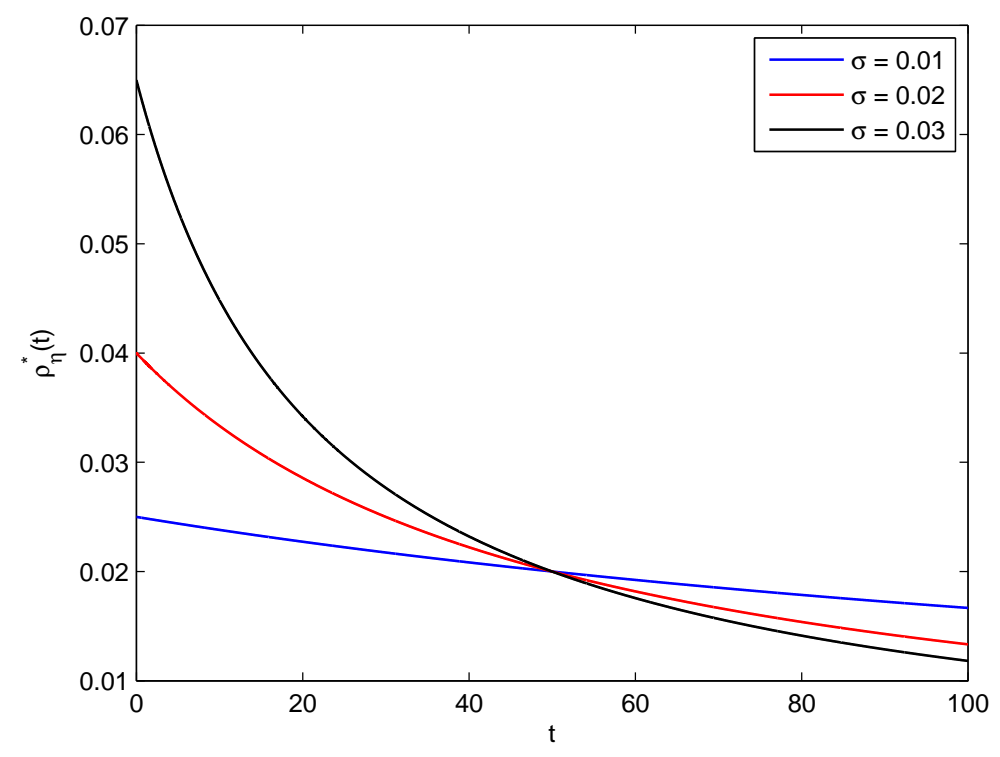

Figure 1: Dependence of the representative discount rate $\rho_{\eta}^{*}(t)$ on the standard deviation $(\sigma)$ of the distribution of individual rates in the population, for iso-elastic felicity functions. $\mu=0.02, \eta=2$ in this example.

\subsubsection{Policy equivalent preferences in a simple growth model}

In order to understand the quantitative dynamics of the policy-equivalent discount rate $\rho^{*}(t)$ for non iso-elastic felicity functions, it is necessary to find the optimal saddle path trajectory of the dynamical system (8-9). This requires numerical methods in general. In order to demonstrate the additional insights that are possible from this exercise, we present the results of such an analysis for a simple model.

We consider a neoclassical growth theory interpretation for our model, in which $S$ is a stock of capital, and $F(S)$ is a Cobb-Douglas production function, minus depreciation. We assume that labour supply is constant (normalized to 1), and no technical progress. Thus,

$$
F(S)=S^{\gamma}-\delta S
$$

We pick $\gamma=0.3$ for the capital share of production, and $\delta=0.1$ for the annual depreciation rate. As we have shown, $T(c)$ must be nonlinear to pick up the additional effects of the endogenous income term $F^{\prime}(S)$ in $(24)$. We pick $T(c)=c^{2}$ - this choice obeys the conditions in Proposition 2. Integrating this choice of $T$ using the relationship (44) in Appendix A yields the marginal felicity function and its inverse, which enter the dynamical equation (9).

We assume that there are two groups of agents in the economy - an impatient group with $\rho=0.04$, and a more patient group with $\rho=0.02$, each with weight $w_{i}=0.5$. We pick an initial condition of $S_{0}=2$, use a shooting method to solve for the initial value of the shadow 


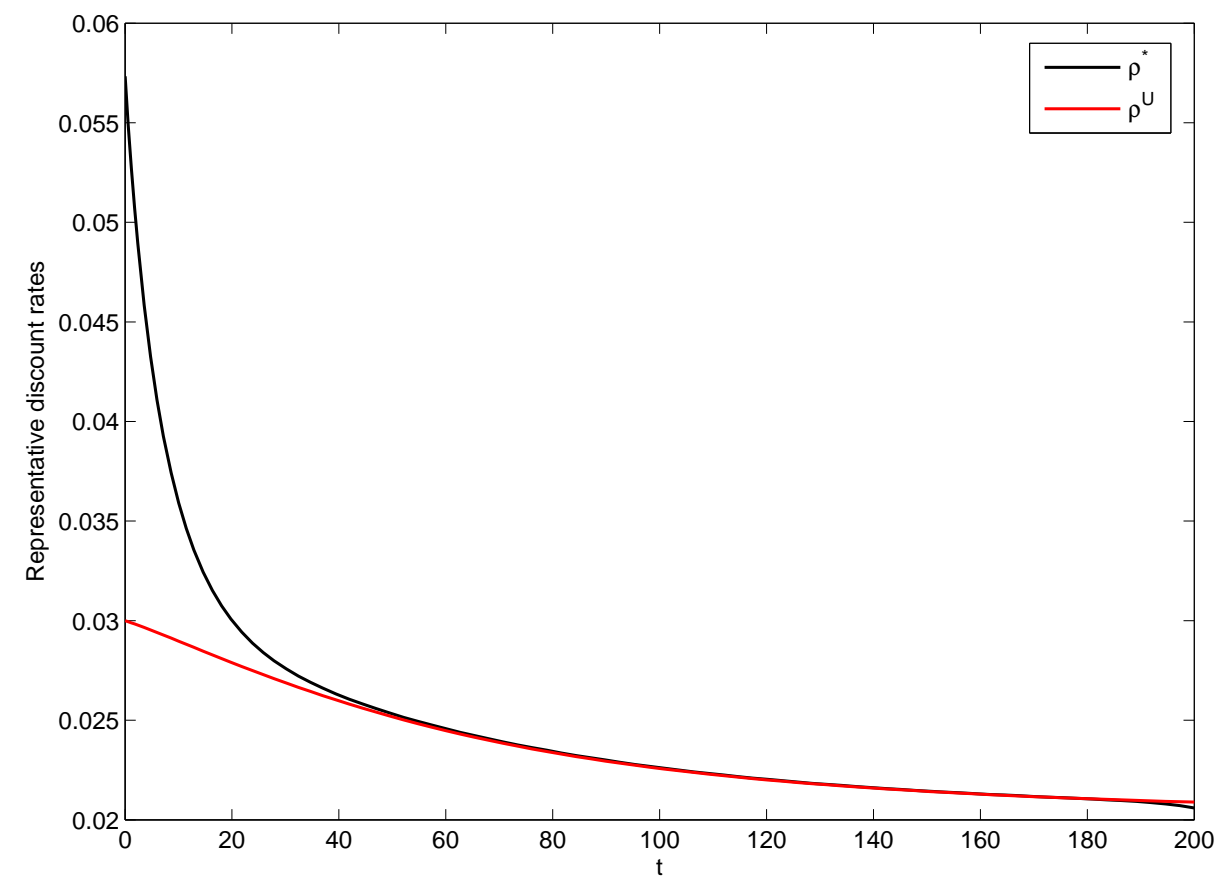

Figure 2: Representative discount rates for our simple growth model (43), and a felicity function with $T(c)=c^{2}$. The black (top) curve gives the term structure of the separable policy equivalent discount rate $\rho^{*}$, and the red (bottom) curve gives the term structure of the welfare equivalent discount rate $\rho^{U}$.

price on the saddle path, and use (24) and (13) to compute the policy and welfare equivalent representative agent's discount rates respectively. Figure 2 contains our results.

By Proposition 2, we know that we must have $\rho^{*}>\rho^{U}$, and that $d \rho^{*} / d t<0$ for this model specification. This is born out in Figure 2. What the simulation results show however is that policy-equivalent and welfare equivalent discount rates can diverge dramatically, especially at early times. The contribution of the first term in (32) to the RA's time preferences is large at early times, as $F^{\prime}(S)$ is a declining function. Figure 2 shows that at early times the policy equivalent discount rate is larger than the discount rate of either group of agents. This is not possible for welfare equivalent preferences, as $\rho^{U}$ is a weighted sum of individual rates, and is thus bounded by the highest and lowest discount rates in the population.

\section{Commitment, time-consistency, and renegotiation proofness}

Throughout the analysis above we have assumed, as is common in the literature on heterogeneous time preferences (e.g. Li and Löfgren, 2000; Gollier and Zeckhauser, 2005), that the social planner can commit to her initial allocations. This assumption may well be met in 
some applications to public decision making, where legal institutions may provide credible commitment devices that constrain policy revisions. If however we relax this assumption, it is clear that the planner's allocation will be time-inconsistent. This is reflected in the fact that representative agents in general do not have constant discount rates, and is known to be a general property of efficient aggregation of heterogeneous time preferences (Jackson and Yariv, 2012) ${ }^{4}$.

The time-inconsistency of the efficient solution raises deep philosophical issues for this approach to public decision-making when no credible commitment devices exist. On the one hand, if the planner is permitted to revise her plan at each point in time, the more impatient agents will receive a much larger share of consumption than was initially agreed upon. Impatience is rewarded, and patience penalized under Pareto efficient revisions. This is a disturbing feature for a normative decision procedure. On the other hand, if we insist on sticking to the initial allocation even at later revision opportunities, one is pressed to ask why $t=0$ social preferences should receive special treatment in deciding allocations at all times.

A non-cooperative approach to this time-inconsistency problem would have us think of the planner as playing a game against "future selves". This approach is familiar from the seminal single agent models of hyperbolic discounting (e.g. Strotz, 1955; Phelps and Pollak, 1968; Laibson, 1997). Markov perfect equilibria of this dynamic game will be time-consistent, but not Pareto efficient. Our view is that this solution, while attractive for behavioral models, is potentially problematic for normative applications with heterogeneous preferences. While in single agent models the dynamic game approach results in an inter-temporal inefficiency, in heterogeneous agent models it also results in distributive inefficiencies, i.e. there will be unexploited opportunities to make the group as a whole better off within a given period, and not simply between periods. Since there is no conceptual or practical difficulty with the standing of agents in intra-generational allocations (as opposed to inter-generational allocations, in which future selves do not have current standing), this seems to remove much of the attractiveness of the efficiency approach as a normative criterion. Indeed, if we are forced to sacrifice distributive efficiency, it is not clear what the normative argument for the weighted-sum social welfare function in equation (1) is.

We suggest that, while the non-cooperative approach suffers from additional difficulties in heterogeneous agent models, the cooperative approach acquires new temporal stability properties that are absent in single-agent models of time-inconsistent choice. In particular, while initial allocations will certainly be time-inconsistent, they are, by virtue of their distributive efficiency, renegotiation proof. Renegotiation proofness is a weaker concept than time-consistency, which may nevertheless provide a strong normative argument for sticking

\footnotetext{
${ }^{4}$ It is possible to choose a different preference aggregation mechanism such as voting, which may under some conditions result in time consistent plans. However, such plans will be inefficient - one is forced to choose between efficiency and consistency, we can't have both. This paper focuses on the efficiency approach, Heal and Millner (2014) considers a consistent voting mechanism, and compares the two approaches
} 
to initial consumption plans. The concept originated as an equilibrium selection mechanism in dynamic games (Farrell and Maskin, 1989; Evans and Maskin, 1989; Abreu and Pearce, 1991). Renegotiation proof contracts are stable under proposals for change at all times - once agreed to, it is not possible to make all players better off by renegotiating the contract at future dates.

The initial allocations in our model are not the equilibria of a non-cooperative dynamic game - we have argued that allowing for such strategic behavior is normatively problematic. Thus the renegotiation proofness concept does not port across wholesale to our setting. However, we can think of the social planner's allocation plan at $t=0$ as a contract between different contemporaneous individuals, and ask whether the group would like to renegotiate this contract at a later date. If the group's initial allocation is efficient with respect to $t=0$ preferences, this will never occur:

Proposition 3. Suppose that the group can agree to a contract that results in efficient dynamic consumption allocations at $t=0$. Then at any future time $\tau>0$, there is no contract the group can agree to that Pareto dominates the initial contract.

Proof. The proof is a trivial consequence of the distributive efficiency of the initial consumption allocations. Suppose (!) that there did exist a revision to the initial plan that made at least one person better off at $t=\tau$, and no one worse off. Then this plan must have been feasible at $t=0$ also, thus violating the efficiency of the initial plan. Hence no such Pareto improving revision can exist.

This simple observation captures the fact that there will always be at least one individual who resists attempts to renegotiate an initially efficient consumption plan. That initial allocations are renegotiation proof, but not time-consistent, is ultimately due to the fact that time-consistency is a 'memoryless' property, whereas renegotiation proofness evaluates proposals for change against a baseline initial contract, and thus retains memory of initially agreed contracts. The losers under a proposed revision remember what was promised to them in the initial contract negotiation, and thus decline welfare reducing revisions to the contract. Since initial plans are efficient, such losers must always exist for any proposed alteration. Thus the presence of multiple heterogeneous agents, rather than a single time-inconsistent planner, lends temporal stability to initially efficient consumption plans ${ }^{5}$.

\section{Conclusions}

While there are many arguments for the use of declining real discount rates for the evaluation of marginal projects (see e.g. the survey in Groom et al. (2005)), the assumption of a

\footnotetext{
${ }^{5}$ Kocherlakota (1996) and Asheim (1997) apply the renegotiation proofness concept in models with a single time-inconsistent agent. Their approach requires us to think of 'future selves' as part of current bargaining processes. In our simple result only current heterogeneous individuals are party to negotiations.
} 
constant pure rate of time preference has remained largely unchallenged in dynamic welfare economics. There are very good reasons for this - the Koopmans axioms make a strong case for the standard exponential discounting model. Despite this, many people are disquieted by the consequences of the exponential discounting model for the evaluation of long-run public policies such as climate change, nuclear waste management, and public infrastructure investments. The exponential decline of the discount factor makes even cataclysmic welfare impacts that are distant in time of very little significance today.

Declining rates of time preference have been proposed as an 'intuitionist' solution to the problem of maintaining the sensitivity of welfare analysis to the long-run effects of policy choices. Building on the work of others, our analysis has shown that such models can be placed on firm normative footing. Representative agent models with declining pure rates of time preference arise naturally when aggregating the preferences of agents with idiosyncratic, time consistent, preferences.

The exact choice of a discount rate schedule for empirical applications is a complex, yet highly policy relevant, task. We have shown that for the purposes of public decisions and cost benefit analysis of public projects, there are two relevant notions of 'representative' preferences. Welfare equivalent preferences capture the group's realized welfare for a given exogenous stream of aggregate consumption, and are in general non-separable. Policy equivalent preferences replicate the group's optimal consumption path, and give rise to the correct sequence of real discount rates. In addition, they are much more flexible than welfare equivalent preferences, and in particular can be chosen to be separable. We have shown that in general policy equivalent preferences depend on agents' idiosyncratic preferences, as well as the productivity of the common resource that generates their income, and must approach the lowest discount rate in the population asymptotically. We demonstrated analytic results for iso-elastic felicity functions, and have argued that under plausible conditions policy equivalent discount rates are monotonically declining. These qualitative properties are shared by the hyperbolic discounting models that have thus far largely been the province of behavioral work. Moreover, we have argued that the dynamic consumption allocations upon which declining representative time preferences are based are renegotiation proof, and thus stable under proposals for change. Our analysis thus suggests that it may be time to elevate models with declining rates of time preference from purely descriptive applications to tools of normative policy analysis.

\section{A Conditions on $T^{i}(c)$}

The condition $\lim _{c \rightarrow 0} U_{i}^{\prime}(c)=\infty$ ensures that solutions to optimal consumption allocation problems are interior. This condition places restrictions on the behavior of $T^{i}(c)$, defined in (2), at the origin. The results in this appendix make use of the following simple lemma: 
Lemma 1. Suppose $A(c)$ is a twice differentiable function. If $\lim _{c \rightarrow 0^{+}} A(c)=-\infty$, then $\lim _{c \rightarrow 0^{+}} A^{\prime}(c)=\infty$.

The proof of this lemma is a simple application of the mean value theorem.

Treating (2) as a differential equation in $U^{\prime}$, we have

$$
U_{i}^{\prime}(c)=M \exp \left(-\int_{0}^{c}\left[T^{i}(x)\right]^{-1} d x\right)
$$

for some constant $M>0$. Define $G(c):=\int_{0}^{c}\left[T^{i}(x)\right]^{-1} d x . \lim _{c \rightarrow 0} U_{i}^{\prime}(c)=\infty$ implies that we require $\lim _{c \rightarrow 0} G(c)=-\infty$. This in turn implies that we must have $\lim _{c \rightarrow 0} G^{\prime}(c)=$ $\lim _{c \rightarrow \infty}\left[T^{i}(c)\right]^{-1}=\infty$, and hence we conclude that

$$
\lim _{c \rightarrow 0} T^{i}(c)=0
$$

The fact that $T^{i}$ approaches zero at the origin means that for $c$ small enough $T^{i}(c) \sim K c^{\alpha}$, for some constants $K, \alpha>0$, where $\alpha$ is the exponent of the dominant term in $T^{i}(c)$ as $c \rightarrow 0$, i.e. all other terms approach zero faster than $c^{\alpha}$. Thus for small $c, G(c) \sim \frac{1}{K(1-\alpha)} c^{1-\alpha}$. Since we need $\lim _{c \rightarrow 0} G(c)=-\infty$, we must have $\alpha \geq 1$. Hence $T^{i}(c)$ must be locally convex at the origin, and approach zero as fast as, or faster than $c$. This in turn implies that $T^{i} / T^{\prime i} \sim c$ as $c \rightarrow 0$, and thus $T^{i} / T^{i}$ is also locally convex at the origin.

\section{B Proof of Proposition 1}

Equations (10) and (8) imply that in the steady state

$$
U_{i}^{\prime}\left(c_{t i}\right) \propto \frac{e^{-\left(F^{\prime}\left(S_{\infty}\right)-\rho_{i}\right) t}}{w_{i}}
$$

where the proportionality constant is positive (since the shadow price is positive). Since $U_{i}^{\prime}$ is decreasing by assumption, this implies that in the steady state $c_{t i}$ is growing indefinitely if $\rho_{i}<F^{\prime}\left(S_{\infty}\right)$, declining if $\rho_{i}>F^{\prime}\left(S_{\infty}\right)$, and constant if $\rho_{i}=F^{\prime}\left(S_{\infty}\right)$. Now in the steady state we require $\dot{S}=0$, i.e. $\sum_{i} c_{t i}=F\left(S_{\infty}\right)$. This cannot be the case if any of the $c_{t i}$ are growing indefinitely. Since the $\rho_{i}$ are distinct, this means that at most one of the $c_{t i}$ can be constant, and the rest must all decline to zero. Clearly, this can only be so if $F^{\prime}\left(S_{\infty}\right)=\rho_{L}$, which implies $\lim _{t \rightarrow \infty} c_{t i}=0$ for $i \neq L$. By the concavity of $F(S)$ there is only one value of $S$ that satisfies $F^{\prime}(S)=\rho_{L}$. Thus this is the unique nontrivial steady state.

To prove that the steady state is a saddle point, from (8-9), the Jacobian of the system is:

$$
J=\left(\begin{array}{ll}
\frac{\partial \dot{\lambda}}{\partial \lambda} & \frac{\partial \dot{\lambda}}{\partial S} \\
\frac{\partial \dot{S}}{\partial \lambda} & \frac{\partial \dot{S}}{\partial S}
\end{array}\right)=\left(\begin{array}{cc}
-F^{\prime}(S) & -\lambda F^{\prime \prime}(S) \\
\sum_{i} T^{i}\left(c_{t i}\right) & F^{\prime}(S)
\end{array}\right)
$$


where in the bottom left element we've used the fact that

$$
\frac{\partial}{\partial \lambda}\left(U_{i}^{\prime}\right)^{-1}\left(e^{\rho_{i} t} \lambda / w_{i}\right)=-\frac{e^{\rho_{i} t} / w_{i}}{U_{i}^{\prime \prime}\left(\left(U_{i}^{\prime}\right)^{-1}\left(e^{\rho_{i} t} \lambda / w_{i}\right)\right)}=T^{i}\left(c_{t i}\right)
$$

Evaluated at the steady state values of $\lambda, S, c_{t i}$, this becomes

$$
\left.J\right|_{S=S_{\infty}}=\left(\begin{array}{cc}
-\rho_{L} & 0 \\
T^{L}\left(F\left(\rho_{L}\right)\right) & \rho_{L}
\end{array}\right)
$$

The eigenvalues of the Jacobian are $\pm \rho_{L}$, and hence the steady state is a saddle point.

Finally, by (27) the steady state discount rate is given by the formula (13). Substituting the steady state consumption values into this formula, and making use of (3), yields (30).

\section{Proof of Proposition 2}

We consider the case in which a non-trivial steady state $S_{\infty}$ exists first, i.e. we assume that $F^{\prime}(S)=\rho_{L}$ has a solution. From $(24)$, we have

$$
\dot{\rho}^{*}=(1-X) F^{\prime \prime}(S) \dot{S}-\dot{X}\left(F^{\prime}(S)-\rho^{U}\right)+X \dot{\rho}^{U}
$$

By the assumption that $T$ is convex, and (3), we know that $T$ must be a super additive function, and hence $0 \leq X \leq 1$. Thus $\operatorname{sgn}(1-X) F^{\prime \prime}(S) \dot{S}=-\operatorname{sgn}(\dot{S})=-\operatorname{sgn}\left(S_{\infty}-S_{0}\right)$, where the last equality follows from the monotonicity of the saddle path. Similarly, from (20) we have $\operatorname{sgn}\left(F^{\prime}(S)-\rho^{U}\right)=\operatorname{sgn}\left(\sum_{i} \dot{c}_{t i}\right)=\operatorname{sgn}\left(S_{\infty}-S_{0}\right)$. Again, the last equality follows from the properties of the saddle path. If $S_{0}<S_{\infty}$, the group's income $F(S)$ is growing along the saddle path. It thus cannot be optimal for aggregate consumption to be falling, and we conclude that $\sum_{i} \dot{c}_{t i}>0$. Thus the crux of the problem is to show that $\dot{X}>0$ and $\dot{\rho}^{U}<0$.

Consider $\dot{\rho}^{U}$. From the definition (13), we have

$$
\begin{aligned}
\dot{\rho}^{U} & =\frac{\left(\sum \rho_{i} T_{i}^{\prime} \dot{c}_{t i}\right) \sum T_{i}-\left(\sum \rho_{i} T_{i}\right) \sum T_{i}^{\prime} \dot{c}_{t i}}{\left(\sum T_{i}\right)^{2}} \\
& =\left(F^{\prime}(S)+\rho^{U}\right) \frac{\sum \rho_{i} T_{i} T_{i}^{\prime}}{\sum T_{i}}-\frac{\sum \rho_{i}^{2} T_{i} T_{i}^{\prime}}{\sum T_{i}}-F^{\prime}(S) \rho^{U} \frac{\sum T_{i}^{\prime} T_{i}}{\sum T_{i}}
\end{aligned}
$$

where $T_{i}:=T\left(c_{t i}\right)$, and we've used (19-20) and simplified. If we set $F^{\prime}(S)=\rho^{U}$ in this equation, we recover the expression for $\dot{\rho}^{U}$ that Gollier and Zeckhauser (2005) obtain in Proposition 5 of their paper. They prove that at this value of $F^{\prime}(S), \dot{\rho}^{U}<0$ if $T^{\prime}>0$. So if we can show that $\left.\dot{\rho}^{U}\right|_{F^{\prime}(S)>\rho^{U}}<\left.\dot{\rho}^{U}\right|_{F^{\prime}(S)=\rho^{U}}$, we will be done. Partially differentiate the 
expression for $\dot{\rho}^{U}$ above with respect to $F^{\prime}(S)$ to find:

$$
\frac{\partial \dot{\rho}^{U}}{\partial F^{\prime}(S)}=\frac{\sum \rho_{i} T_{i} T_{i}^{\prime}}{\sum T_{i}}-\frac{\sum \rho_{i} T_{i}}{\sum T_{i}} \frac{\sum T_{i} T_{i}^{\prime}}{\sum T_{i}}
$$

where we've used $\rho^{U}=\left(\sum \rho_{i} T_{i}\right) /\left(\sum T_{i}\right)$. Define the expectation operator, $\left\langle x_{i}\right\rangle:=\frac{\sum x_{i} T_{i}}{\sum T_{i}}$. Then the expression above is,

$$
\begin{aligned}
\frac{\partial \dot{\rho}^{U}}{\partial F^{\prime}(S)} & =\left\langle\rho_{i} T_{i}^{\prime}\right\rangle-\left\langle\rho_{i}\right\rangle\left\langle T_{i}^{\prime}\right\rangle \\
& =\operatorname{Cov}\left(\rho_{i}, T^{\prime}\left(c_{t i}\right)\right)
\end{aligned}
$$

where $\operatorname{Cov}(x, y)$ is the covariance of the two random variables $x, y$. Now from (10) it's clear that

$$
\frac{\partial c_{t i}}{\partial \rho_{i}}=-t T\left(c_{t i}\right)<0
$$

Hence $\rho_{i}$ and $c_{t i}$ are anti-correlated, and when $T^{\prime}$ is an increasing function, $\operatorname{Cov}\left(\rho_{i}, T^{\prime}\left(c_{t i}\right)\right)<$ $0 \Rightarrow \frac{\partial \dot{\rho}^{U}}{\partial F^{\prime}(S)}<0$. Hence for $F^{\prime}(S)>\rho^{U}$ this implies that $\dot{\rho}^{U}<\left.\dot{\rho}^{U}\right|_{F^{\prime}(S)=\rho^{U}}<0$.

Now consider $\dot{X}$. Differentiating $X$ directly using the definition (23), we see that $\dot{X}>0$ iff

$$
\begin{aligned}
& \left(\sum_{i} T_{i}^{\prime} \dot{c}_{t i}\right) T\left(\sum_{i} c_{t i}\right)>\left(\sum T_{i}\right) T^{\prime}\left(\sum_{i} c_{t i}\right) \sum_{i} \dot{c}_{t i} \\
\Longleftrightarrow & \left(\sum_{i} T_{i}^{\prime} T_{i}\left(F^{\prime}(S)-\rho_{i}\right)\right) T\left(\sum_{i} c_{t i}\right)>T^{\prime}\left(\sum_{i} c_{t i}\right)\left(\sum T_{i}\right)^{2}\left(F^{\prime}(S)-\rho^{U}\right) \\
\Longleftrightarrow & \frac{\left(\sum_{i} T_{i}^{\prime} T_{i}\left(F^{\prime}(S)-\rho_{i}\right)\right)}{\sum T_{i}}-\frac{T^{\prime}\left(\sum c_{t i}\right)}{T\left(\sum c_{t i}\right)}\left(\sum T_{i}\right)\left(F^{\prime}(S)-\rho^{U}\right)>0
\end{aligned}
$$

where in the second line we've used the expressions for $\dot{c}_{t i}$ and $\sum_{i} \dot{c}_{t i}$ in (19-20). Consider the first term:

$$
\begin{aligned}
\frac{\left(\sum_{i} T_{i}^{\prime} T_{i}\left(F^{\prime}(S)-\rho_{i}\right)\right)}{\sum T_{i}} & =F^{\prime}(S)\left\langle T_{i}^{\prime}\right\rangle-\left\langle\rho_{i} T_{i}^{\prime}\right\rangle \\
& =F^{\prime}(S)\left\langle T_{i}^{\prime}\right\rangle-\left[\operatorname{Cov}\left(\rho_{i}, T_{i}^{\prime}\right)+\left\langle T_{i}^{\prime}\right\rangle\left\langle\rho_{i}\right\rangle\right] \\
& =-\operatorname{Cov}\left(\rho_{i}, T_{i}^{\prime}\right)+\left(F^{\prime}(s)-\rho^{U}\right)\left\langle T_{i}^{\prime}\right\rangle
\end{aligned}
$$

Hence $\dot{X}>0$ iff

$$
-\operatorname{Cov}\left(\rho_{i}, T_{i}^{\prime}\right)+\left(F^{\prime}(S)-\rho^{U}\right)\left[\frac{\sum_{i} T_{i}^{\prime} T_{i}}{\sum T_{i}}-\frac{T^{\prime}\left(\sum c_{t i}\right)}{T\left(\sum c_{t i}\right)}\left(\sum T_{i}\right)\right]>0
$$

We have shown that when $T$ is convex, $-\operatorname{Cov}\left(\rho_{i}, T_{i}^{\prime}\right)>0$, and we also have $F^{\prime}(S)-\rho^{U}>0$ on 
the saddle path, so the task is to find conditions under which the square bracket is positive. Rewrite this condition as:

$$
\frac{T\left(\sum c_{t i}\right)}{T^{\prime}\left(\sum c_{t i}\right)} \sum_{i} T_{i}^{\prime} T_{i}>\left(\sum T_{i}\right)^{2}
$$

Now assume that $T / T^{\prime}$ is a convex function. This, in combination with the fact that $\lim _{c \rightarrow 0} T(c)=0$, implies that $\lim _{c \rightarrow 0} T / T^{\prime}=0$, and hence $T / T^{\prime}$ is super additive. Thus,

$$
\frac{T\left(\sum c_{t i}\right)}{T^{\prime}\left(\sum c_{t i}\right)} \geq \sum_{j} \frac{T_{j}}{T_{j}^{\prime}}
$$

Under this condition, it is sufficient to show that

$$
\begin{aligned}
& \left(\sum_{i} T_{i}^{\prime} T_{i}\right)\left(\sum_{j} \frac{T_{j}}{T_{j}^{\prime}}\right)>\left(\sum T_{i}\right)^{2}=\sum_{i} T_{i} \sum_{j} T_{j} \\
& \Longleftrightarrow \sum_{i} \sum_{j} T_{i} T_{j}\left(\frac{T_{i}^{\prime}}{T_{j}^{\prime}}-1\right)>0
\end{aligned}
$$

Notice that when $i=j$ in this double sum the factor in brackets is zero. Also, the factor $T_{i} T_{j}$ is symmetric under $i \leftrightarrow j$, so we can factorize pairs of terms with symmetric indices to find that we require

$$
\sum_{i} \sum_{j>i} T_{i} T_{j}\left(\frac{T_{i}^{\prime}}{T_{j}^{\prime}}+\frac{T_{j}^{\prime}}{T_{i}^{\prime}}-2\right)>0 .
$$

The factors in the round brackets are all of the form $x+x^{-1}-2$, where $x \geq 0$. It is easy to show that this function has a global minimum at $x=1$, at which its value is zero. Hence the double sum is non-negative, and $\dot{X}>0$. Combining these pieces yields $\dot{\rho}^{*}<0$.

Now consider the case of exhaustible resources, in which $F(S)=0$. Clearly the equation $F^{\prime}(S)=\rho_{L}$ has no solution in this case, so there is no non-trivial steady state, and this case is not covered by the derivation above. Since $F^{\prime}(S)=0$ in this case, we have from our expression (24) that

$$
\rho^{*}(t)=\frac{\sum_{i} \rho_{i} T\left(c_{t i}\right)}{T\left(\sum_{i} c_{t i}\right)}
$$


Differentiate $\rho^{*}(t)$ explicitly with respect to time to find that $\operatorname{sgn} \dot{\rho}^{*}(t)$ is given by the sign of

$$
\begin{aligned}
& \left(\sum_{i} \rho_{i} T^{\prime}\left(c_{t i}\right) \dot{c}_{t i}\right) T\left(\sum_{i} c_{t i}\right)-\left(\sum_{i} \rho_{i} T\left(c_{t i}\right)\right) T^{\prime}\left(\sum_{i} c_{t i}\right) \sum_{i} \dot{c}_{t i} \\
& =\sum_{i}\left[\rho_{i} T^{\prime}\left(c_{t i}\right) T\left(\sum_{i} c_{t i}\right)-\left(\sum_{i} \rho_{i} T\left(c_{t i}\right)\right) T^{\prime}\left(\sum_{i} c_{t i}\right)\right] \dot{c}_{t i} \\
& =-T^{\prime}\left(\sum_{i} c_{i t}\right)\left[\left(\sum_{i} \rho_{i}^{2} T_{i} T_{i}^{\prime}\right) \frac{T\left(\sum_{i} c_{t i}\right)}{T^{\prime}\left(\sum c_{t i}\right)}-\left(\sum_{i} \rho_{i} T_{i}\right)^{2}\right]
\end{aligned}
$$

where we've used $\dot{c}_{t i}=-\rho_{i} T\left(c_{t i}\right)$ in the third line. Now consider the factor in the square bracket, and assume $T / T^{\prime}$ is convex, so that (59) holds. Then to show that this factor is non-negative it is sufficient to show that

$$
\begin{aligned}
& \left(\sum_{i} \rho_{i}^{2} T_{i} T_{i}^{\prime}\right)\left(\sum_{j} \frac{T_{j}}{T_{j}^{\prime}}\right)-\left(\sum_{i} \rho_{i} T_{i}\right)^{2}>0 \\
& \Longleftrightarrow \sum_{i} \sum_{j}\left(\rho_{i}^{2} T_{i} T_{j} \frac{T_{i}^{\prime}}{T_{j}^{\prime}}-\rho_{i} \rho_{j} T_{i} T_{j}\right)>0 \\
& \Longleftrightarrow \sum_{i} \sum_{j} \rho_{i} \rho_{j} T_{i} T_{j}\left(\frac{\rho_{i}}{\rho_{j}} \frac{T_{i}^{\prime}}{T_{j}^{\prime}}-1\right)>0 \\
& \Longleftrightarrow \sum_{i} \sum_{j>i} \rho_{i} \rho_{j} T_{i} T_{j}\left(\frac{\rho_{i}}{\rho_{j}} \frac{T_{i}^{\prime}}{T_{j}^{\prime}}+\frac{\rho_{j}}{\rho_{i}} \frac{T_{j}^{\prime}}{T_{i}^{\prime}}-2\right)>0
\end{aligned}
$$

Again, the factors in brackets are of the form $x+x^{-1}-2$, and are thus positive. Hence $\dot{\rho}^{*}<0$ in the exhaustible resources case as well.

Finally, from (24) and (20), we have

$$
\begin{aligned}
\rho^{*}-\rho^{U} & =(1-X)\left(F^{\prime}(S)-\rho^{U}\right) \\
& =(1-X) \frac{\sum_{i} \dot{c}_{t i}}{\sum_{i} T\left(c_{t i}\right)}
\end{aligned}
$$

Recall that $0<X<1$ when $T$ is convex. If there is a non-trivial steady state, $\operatorname{sgn}\left(\sum_{i} \dot{c}_{t i}\right)=$ $\operatorname{sgn}\left(S_{\infty}-S_{0}\right)$, and we conclude that $\rho^{*} \geq \rho^{U}$. For exhaustible resources $F^{\prime}(S)=0$ and we find that $\rho^{U} \geq \rho^{*}$.

\section{References}

Abreu, Dilip, and David Pearce. 1991. "A Perspective on Renegotiation in Repeated Games." In Game Equilibrium Models II. , ed. Professor Dr Reinhard Selten, 44-55. 
Springer Berlin Heidelberg.

Arrow, Kenneth J. 1999. "Discounting, morality, and gaming." In Discounting and Intergenerational Equity. , ed. Paul R. Portney and John P. Weyant. Resources for the Future.

Asheim, Geir B. 1997. "Individual and Collective Time-Consistency." The Review of Economic Studies, 64(3): 427-443.

Barro, Robert J. 1999. "Ramsey Meets Laibson in the Neoclassical Growth Model." The Quarterly Journal of Economics, 114(4): 1125-1152.

Becker, Robert A. 1980. "On the Long-Run Steady State in a Simple Dynamic Model of Equilibrium with Heterogeneous Households." The Quarterly Journal of Economics, 95(2): 375-382.

Cvitanić, Jakša, Elyès Jouini, Semyon Malamud, and Clotilde Napp. 2012. "Financial Markets Equilibrium with Heterogeneous Agents." Review of Finance, 16(1): 285-321.

Dasgupta, Partha. 2012. "Time and the generations." In Climate Change and Common Sense: Essays in Honor of Tom Schelling. , ed. Robert W. Hahn and Alistair Ulph. Oxford University Press.

Dasgupta, Partha, and Eric Maskin. 2005. "Uncertainty and Hyperbolic Discounting." American Economic Review, 95(4): 1290-1299.

Dasgupta, P. S., and G. M. Heal. 1979. Economic Theory and Exhaustible Resources. Cambridge University Press.

Dumas, B. 1989. "Two-person dynamic equilibrium in the capital market." Review of Financial Studies, 2(2): 157-188.

Evans, Robert, and Eric Maskin. 1989. "Efficient renegotiation-proof equilibria in repeated games." Games and Economic Behavior, 1(4): 361-369.

Farrell, Joseph, and Eric Maskin. 1989. "Renegotiation in repeated games." Games and Economic Behavior, 1(4): 327-360.

Feldstein, M. S. 1964. "The Social Time Preference Discount Rate in Cost Benefit Analysis." The Economic Journal, 74(294): 360.

Frederick, Shane, George Loewenstein, and Ted O'Donoghue. 2002. "Time Discounting and Time Preference: A Critical Review." Journal of Economic Literature, 40(2): 351401.

Gollier, Christian, and Richard Zeckhauser. 2005. "Aggregation of Heterogeneous Time Preferences." Journal of Political Economy, 113(4): 878-896. 
Groom, Ben, Cameron Hepburn, Phoebe Koundouri, and David Pearce. 2005. "Declining Discount Rates: The Long and the Short of it." Environmental and Resource Economics, 32(4): 445-493.

Hara, Chiaki. 2009. "Heterogeneous impatience in a continuous-time model." Mathematics and Financial Economics, 2(2): 129-149.

Heal, Geoffrey. 2005. "Intertemporal Welfare Economics and the Environment." In Handbook of Environmental Economics. Vol. 3, , ed. Karl-Göran Mäler and Jeffrey R. Vincent, 1105-1145. Elsevier.

Heal, Geoffrey, and Antony Millner. 2014. "Discounting and Public Choice." In preparation.

Jackson, Matthew O., and Leeat Yariv. 2012. "Collective Dynamic Choice: The necessity of time inconsistency." Working paper.

Jouini, Elyès, Jean-Michel Marin, and Clotilde Napp. 2010. "Discounting and divergence of opinion." Journal of Economic Theory, 145(2): 830-859.

Kamien, Morton I., and Nancy L. Schwartz. 1991. Dynamic Optimization: The Calculus of Variations and Optimal Control in Economics and Management. . 2 ed., Elsevier Science.

Kocherlakota, Narayana R. 1996. "Reconsideration-Proofness: A Refinement for Infinite Horizon Time Inconsistency." Games and Economic Behavior, 15(1): 33-54.

Koopmans, Tjalling C. 1960. "Stationary Ordinal Utility and Impatience." Econometrica, 28(2): 287-309.

Koopmans, Tjalling C. 1969. "Objectives, Constraints, and Outcomes in Optimal Growth Models." In Economic Models, Estimation and Risk Programming: Essays in Honor of Gerhard Tintner. , ed. Karl A. Fox, Jati K. Sengupta and G. V. L. Narasimham, 110-132. Springer Berlin Heidelberg.

Laibson, David. 1997. "Golden Eggs and Hyperbolic Discounting." The Quarterly Journal of Economics, 112(2): 443-478.

Lengwiler, Yvan. 2005. "Heterogeneous Patience and the Term Structure of Real Interest Rates." The American Economic Review, 95(3): 890-896.

Li, Chuan-Zhong, and Karl-Gustaf Löfgren. 2000. "Renewable Resources and Economic Sustainability: A Dynamic Analysis with Heterogeneous Time Preferences." Journal of Environmental Economics and Management, 40(3): 236-250. 
Loewenstein, George, and Drazen Prelec. 1992. "Anomalies in Intertemporal Choice: Evidence and an Interpretation." The Quarterly Journal of Economics, 107(2): 573-597.

Nocetti, Diego, Elyès Jouini, and Clotilde Napp. 2008. "Properties of the Social Discount Rate in a Benthamite Framework with Heterogeneous Degrees of Impatience." Management Science, 54(10): 1822-1826.

Nordhaus, William D. 2007. "A Review of the Stern Review on the Economics of Climate Change." Journal of Economic Literature, 45(3): 686-702.

Phelps, E. S., and R. A. Pollak. 1968. "On Second-Best National Saving and GameEquilibrium Growth." The Review of Economic Studies, 35(2): 185-199.

Stern, N. H. 2007. The economics of climate change : the Stern review. Cambridge:Cambridge University Press.

Strotz, R. H. 1955. "Myopia and Inconsistency in Dynamic Utility Maximization." The Review of Economic Studies, 23(3): 165.

Weitzman, Martin L. 1998. "Why the Far-Distant Future Should Be Discounted at Its Lowest Possible Rate,." Journal of Environmental Economics and Management, 36(3): 201 $-208$.

Weitzman, Martin L. 2001. "Gamma Discounting." The American Economic Review, 91(1): 260-271.

Weitzman, Martin L. 2007. "A Review of The Stern Review on the Economics of Climate Change." Journal of Economic Literature, 45(3): 703-724.

Wilson, Robert. 1968. "The Theory of Syndicates." Econometrica, 36(1): 119. 